\title{
A young and complex binary star - HD 144432
}

\author{
M. R. Pérez ${ }^{1}$, M. E. van den Ancker ${ }^{2, \star, \star \star}$, D. de Winter ${ }^{3}$, and B. W. Bopp ${ }^{4}$ \\ 1 Los Alamos National Laboratory, PO Box 1663, ISR-DO, Mail Stop F650, Los Alamos, NM 87545, USA \\ 2 Harvard-Smithsonian Center for Astrophysics, 60 Garden Street, MS 42, Cambridge, MA 02138, USA \\ e-mail: mvandena@eso.org \\ 3 TNO-TPD, Stieltjesweg 1, PO Box 155, 2600 AD Delft, The Netherlands \\ e-mail: dolf@xiada.ft.uam.es \\ 4 The University of Toledo, Dept. of Physics and Astronomy, Toledo, OH 43606, USA \\ e-mail: bbopp@uoftQ2 .utoledo.edu
}

Received 24 September 2003 / Accepted 17 November 2003

\begin{abstract}
The southern emission-line star HD 144432 has received considerable attention due to its relative brightness $(V \sim 8.17)$, its late spectral type (late A-type or perhaps early F) and its relative isolation from a bona-fide active star formation region. We present new imaging and spectroscopic data of this star, which in the past has been classified as both an evolved (post-AGB) object and an isolated Herbig Ae/Be star. We confirm the presence of a faint companion source located $1.4^{\prime \prime}$ north, which appears physically associated with HD 144432. New infrared spectroscopy reveals this companion to be a late-type (early-mid K) star, devoid of any emission lines. Furthermore, we confirm the pre-main sequence nature of this object, report the detection of Li I $6707.8 \AA$ absorption toward the HD 144432 system, and its apparent association with Sco OB2-2 located at $145 \mathrm{pc}$.
\end{abstract}

Key words. stars: circumstellar matter - stars: emission-line, Be - stars: individual: HD 144432 - stars: binaries: visual stars: pre-main sequence

\section{Introduction}

The star HD 144432 (SAO 184124, He 3-1141, IRAS 16038-2735) is one the latest in spectral type among the Herbig $\mathrm{Ae} / \mathrm{Be}(\mathrm{HAeBe})$ stars and candidates listed by Thé et al. (1994). These late-type Herbig star have recently spurred some interest, since there appears to be a pronounced absence of emission-line stars with late A or early $\mathrm{F}$ spectral types within the group of HAeBe stars. Since Herbig (1960), in the descriptive paper of the class, restricted his analysis to Beand Ae-type stars, this was thought to be primarily for historic reasons. However, it has proven to be difficult to find additional young objects among the F-type stars. Suchkov et al. (2002) from a sample of about 900 metal-rich reddened F stars, found that about 70 stars presented abnormal near-infrared color excesses, suggesting the presence of circumstellar material. However, they listed only 21 selected stars from the original

Send offprint requests to: M. R. Pérez,

e-mail: mperez@lanl.gov

* Now at European Southern Observatory, Karl-SchwarzschildStrasse 2, 85748, Garching bei München, Germany.

$\star \star$ Visiting Astronomer at the Infrared Telescope Facility, which is operated by the University of Hawaii under Cooperative Agreement no. NCC 5-538 with the National Aeronautics and Space Administration, Office of Space Science, Planetary Astronomy Program. large sample; HD 144432 was included among the several "re-discovered" objects.

HD 144432 was described as an IRAS point source by Oudmaijer et al. (1992), with the note that this object could be either a post AGB or an Ae Herbig star. Based on the IRAS colors, Oudmaijer et al. (1992) were able to derive a color or dust temperature of $240 \mathrm{~K}$, by means of a black body fit to the excess fluxes.

Likewise, Carballo et al. (1992), using IRAS fluxes, described HD 144432 as part of the Scorpio-Centaurus-Lupus region. However, they classified it as an evolved galactic object of spectral type FOIIIe, with a dust shell signature due to mass loss. Furthermore, these authors commented that this object was suggested to be a binary by Wackerling (1970) and that Walker \& Wolstencroft (1988) erroneously classified it as a Vega-type star. However, in their catalogue of HaeBe stars, Thé et al. (1994) considered HD 144432 to be a probable member of this stellar group; a classification followed by most subsequent authors.

Motivated by the list of Vega-type stars by Walker \& Wolstencroft (1988), Walker \& Butner (1995) observed HD 144432, among several other objects, with $10 \mu \mathrm{m}$ spectroscopy and sub-mm observations, finding that the stars in their sample show strong emission features due to silicate dust. Similarly, Sylvester et al. (1995) reported a broad emission feature at $9.7 \mu \mathrm{m}$ and possibly at $18 \mu \mathrm{m}$, indicating the presence 
of silicate dust. These authors also pointed out that the excess emissions seen at long wavelengths, in HD 144432 and other objects, have spectral slopes shallower than those predicted for Rayleigh-Jeans emission, suggesting the presence of large grains (radius $\geq 0.1 \mathrm{~mm}$ ) and large amounts of cool material.

By studying a sample of stars surrounded by dust, which included HD 144432, Zuckerman et al. (1995) concluded that for stars with ages $10^{6}-10^{7}$ years, the gas and dust are well mixed and that this gas dissipates rapidly implying that if gas-giant planets (e.g., Jupiter, Saturn) are common in planetary systems, they must form more quickly than present models indicate.

Recently, Maheswar et al. (2002) listed HD 144432 among the objects studied in search of an inconclusive geometrical relationship between binary position angle, polarization position angle and outflow position angle. Further infrared observations including HD 144432 as one the sources studied at $10 \mu \mathrm{m}$ by van Boekel et al. (2003), found a correlation between the strength of the silicate emission, its shape and the local continuum, which is interpreted as the evidence for removal of small grains $(0.1 \mu \mathrm{m})$, whereas large grains $(1-2 \mu \mathrm{m})$ remain on the disk surface.

In this paper, we present unpublished observations of the emission line object HD 144432, and we analyze some possible scenarios of its current status and evolution.

\section{Observations}

\subsection{Optical and near-IR imaging}

Deep CCD images of HD 144432 through Bessell R, Gunn i, and narrowband $\mathrm{H} \alpha$, [O III] and [Si II] filters were obtained, under good seeing conditions, with the Dutch $92 \mathrm{~cm}$ telescope at the European Southern Observatory (ESO)- La Silla, Chile on July 29, 1994. The total field of view in these images was $4^{\prime} \times 4^{\prime}$. Since the main purpose of these images was to look for extended, faint nebulosity in the area surrounding HD 144432, the few arcseconds in the immediate vicinity of HD 144432 were saturated in all images. No traces of extended nebulous structure or a dark region could be seen in any of the images, nor were there any anomalies in the relative strength in the various filters of the different sources in the field of view. From these images we conclude that HD 144432 is apparently an isolated star, not directly associated with a clearly identifiable star-forming region, or a group of fainter emission-line stars.

Recent $K(2.2 \mu \mathrm{m})$ band imaging secured at the $3.0 \mathrm{~m}$ NASA Infrared Telescope Facility (IRTF), reveals a faint second source (about 2.4 fainter in $K$ than the primary), located $1.4^{\prime \prime}$ north (position angle $-7^{\circ}$ ). In the remainder of this paper, when distinguishing individual components is possible, we refer to the southernmost, IR-brightest component as HD 144432 A and the fainter, northernmost of the two, as HD 144432 B. In Sect. 3 we argue that this secondary is physically associated with HD $144432 \mathrm{~A}$, and we will discuss its properties. A blow-up of the IRTF $K$-band image is shown in Fig. 1 . No other strong $K$-band sources were found in the immediate vicinity of HD 144432, confirming the earlier conclusion that the system appears to be rather isolated.

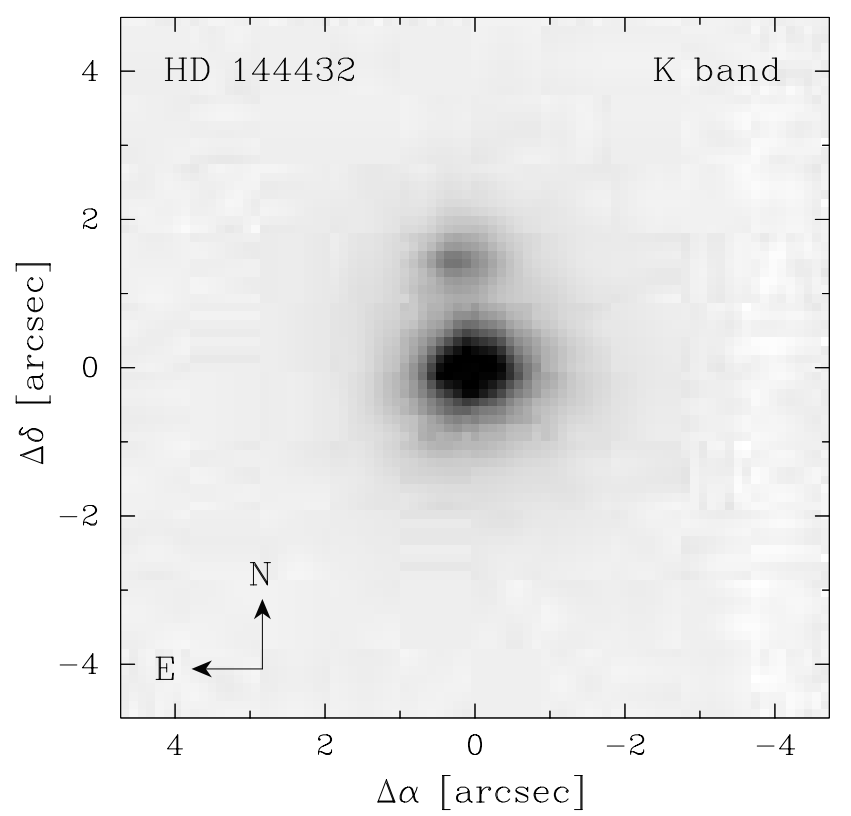

Fig. 1. $K$-band $(2.2 \mu \mathrm{m})$ image of HD 144432, showing the faint binary companion (HD 144432 B) located 1.4" mostly north from HD 144432 A.

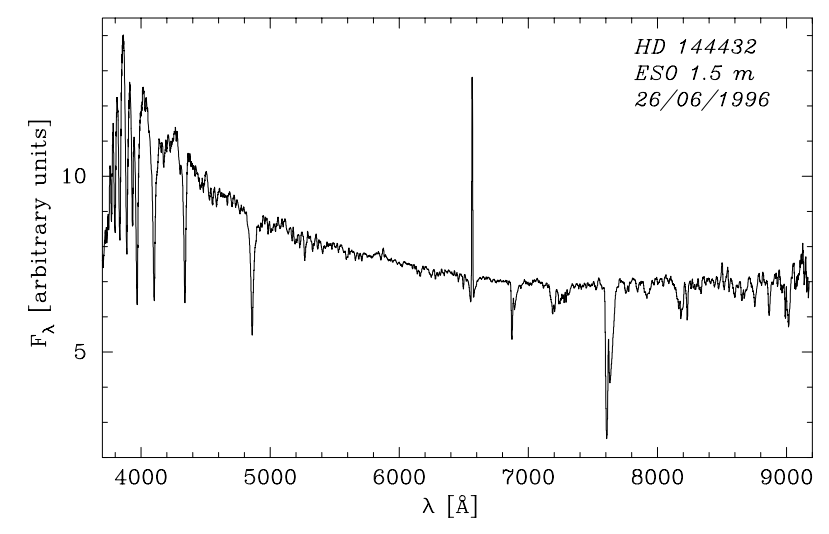

Fig. 2. Optical spectra showing a strong $\mathrm{H} \alpha$, He I (5876 ̊), and Ca II triplet in emission. Continuum flux flattened out toward longer wavelengths illustrates the onset of the near-IR excess.

\subsection{Optical spectroscopy and Li I detection}

A low-resolution (2.83 $\AA$ pixel $\left.^{-1}\right)$ CCD spectrum of HD 144432, covering the wavelength range of 3380 to $9180 \AA$, was obtained on June 27, 1996, using the Boller \& Chivens spectrograph mounted on the ESO $1.5 \mathrm{~m}$ telescope at La Silla, Chile. A slit with a width of $2^{\prime \prime}$ was used to remove the light of other parts of the sky. The spectrum was reduced in a standard fashion using MIDAS. Sky subtraction was achieved by subtracting a third degree polynomial fitted to 100 pixels (corresponding to roughly $25^{\prime \prime}$ ) in the spatial direction on both sides of the stellar spectrum. The reduced spectrum is shown in Fig. 2.

Medium resolution $(\Delta \lambda=0.2 \AA)$ spectroscopic observations were carried out at the KPNO and at Ritter Observatory, University of Toledo, at three spectral settings centered at 6430 , 6750 and $8600 \AA$, respectively. In the first spectral region presented in Fig. 3, metal lines in the spectrum of HD 144432 


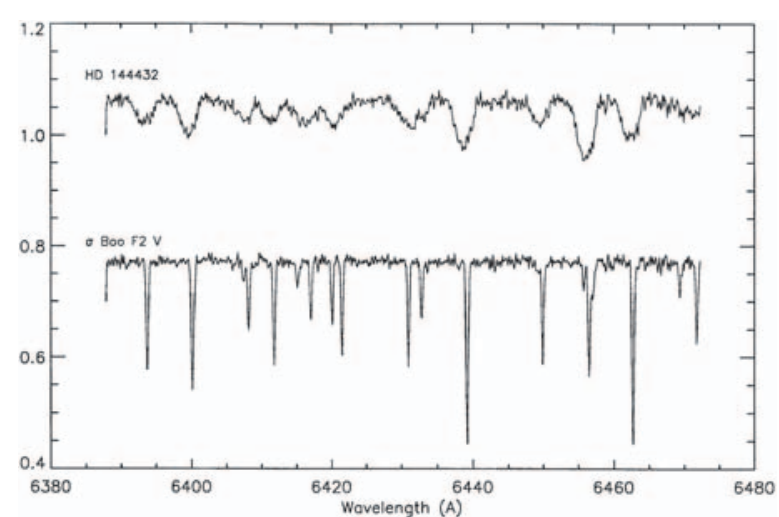

Fig. 3. Metal lines in absorption compared with the main sequence $\sigma$ Boo $(\mathrm{F} 2 \mathrm{~V})$ star. The derived rotational velocity, $v \sin i$, is $72 \pm 5 \mathrm{~km} \mathrm{~s}^{-1}$.

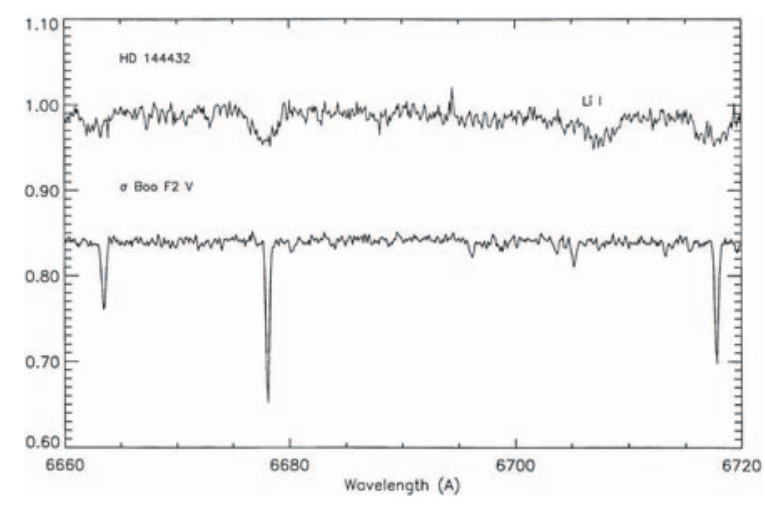

Fig. 4. Lithium absorption present in HD 144432, along with the comparison star $\sigma$ Boo (F2V). Also note that Ca I $6717 \AA$ also appears in absorption.

and $\sigma$ Boo $(\mathrm{F} 2 \mathrm{~V})$ are compared. Based on the broadening of the observed lines $v \sin i$ is derived to be $72 \pm 5 \mathrm{~km} \mathrm{~s}^{-1}$. We note that a subtle indication of asymmetry in the photospheric lines appears to be present but could not be confirmed at the resolutions presented here.

In the second spectral region it is possible to unequivocally detect, for the first time, the absorption at Li I $6707.8 \AA$ in the spectrum of HD 144432. In Fig. 4, we present the spectral comparison of the narrow lines $\sigma$ Boo and HD 144432. We also note that CaI $6717 \AA$ appears in absorption. Both absorption lines could suggest the presence of a cooler companion (Corporon \& Lagrange 1999), but this suggestion is problematic as is discussed in the conclusions section. The equivalent width of the Li I line is $93 \mathrm{~m} \AA$, while the nearby Fe I line $6677 \AA$ is also $93 \mathrm{~m} \AA$, both lines are visible in Fig. 4. This equivalent width of the Li I line is typical of the solar-type stars in the Pleiades which are much older objects $\left(\sim 10^{7}\right.$ years $)$ with significantly lower effective temperatures (e.g., Soderblom et al. 1993).

Optical observations were also made of the $\mathrm{H} \alpha$ emission. Figure 5 presents the P Cygni profiles of HD 144432 taken in two different seasons, showing a variable blue emission component with a variable terminal velocity of $\sim 300-400 \mathrm{~km} \mathrm{~s}^{-1}$. This strong blueshifted emission, present at both epochs,

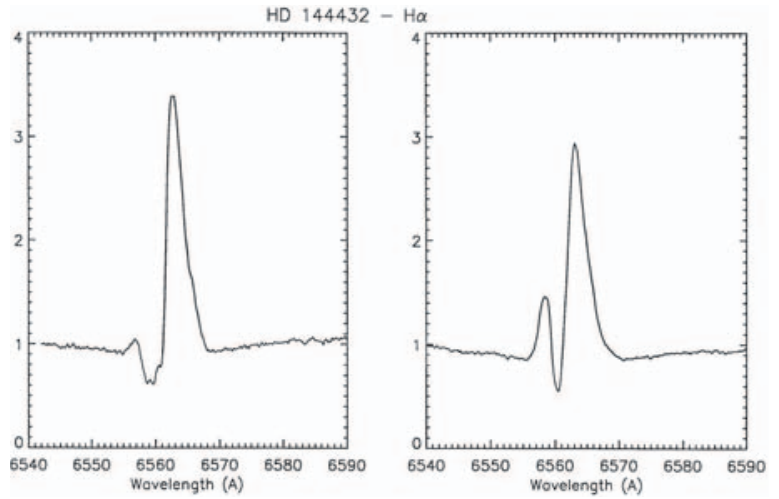

Fig. 5. P Cygni $\mathrm{H} \alpha$ emission presents season-to-season variability common in PMS objects. In contrast, photometric variability is small $(\Delta V<0.05 \mathrm{mag})$.

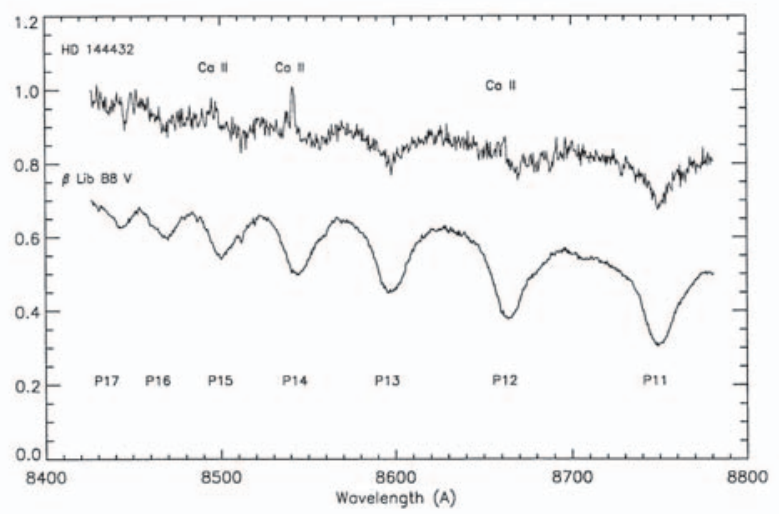

Fig. 6. Ca II triplet emissions are detected in HD 144432. These lines are the signature of a shell, frequent in Be stars, but rarely present in stars later than A0. Comparison star $\beta$ Lib presents none of these emission, however, illustrates the presence of the Paschen absorption lines from P11 to P17.

strengthens the PMS origin of this star by ruling out the possible post-AGB phase since fast winds of this kind are not observed in these stars.

In the region centered at $8600 \AA$, one can detect the presence of the Ca II infrared triplet (IRT) in emission. There are also weak Paschen absorption lines present. In Fig. 6 we have plotted the spectrum of the B8V star $\beta$ Lib to show where the Paschen wavelengths are; we do not suggest that the spectrum of HD 144432 resembles a B8V. The Ca II IRT in emission is a known signature of a shell (e.g., Jaschek et al. 1991), often present in Be stars, but beyond A0 no Ca II emission is seen, except for the alleged peculiar and young stars HD 31648 and 17 Lep (Jaschek et al. 1988), but this is a well-known characteristic of HAeBe stars, even for objects later than A0 (e.g., Catala et al. 1986; Hamann \& Persson 1992). Therefore, the presence of the Ca II IRT in emission in a A9/F0 star is peculiar but not unusual in PMS objects.

\subsection{Ultraviolet spectroscopy}

HD 144432 was observed with the International Ultraviolet Explorer (IUE) satellite on several occasions in 1990 and 
Table 1. IUE Observations of HD 144432.

\begin{tabular}{|c|c|c|c|c|}
\hline $\begin{array}{l}\text { Image } \\
\text { number }\end{array}$ & Date & $\begin{array}{l}\text { Exp. } \\
\text { time }\end{array}$ & $\begin{array}{c}V \\
(\mathrm{FES})\end{array}$ & Comment \\
\hline \multicolumn{5}{|l|}{ SWP } \\
\hline 38425 & 23 Mar. 90 & $30 \mathrm{~min}$ & 8.28 & optimum \\
\hline 39496 & 20 Aug. 90 & $30 \mathrm{~min}$ & 8.32 & optimum \\
\hline 54311 & 06 Apr. 95 & $15 \mathrm{~min}$ & - & underexposed \\
\hline \multicolumn{5}{|l|}{ LWP } \\
\hline 17585 & 23 Mar. 90 & $10 \mathrm{~min}$ & 8.29 & over. $(\sim 3 X)$ \\
\hline 17586 & 23 Mar. 90 & $3 \min$ & 8.31 & optimum \\
\hline 30188 & 09 Mar. 95 & $35 \mathrm{~min}$ & - & overexposed \\
\hline 30354 & 31 Mar. 95 & $10 \mathrm{~min}$ & - & overexposed \\
\hline 30393 & 06 Apr. 95 & $63 \mathrm{~min}$ & - & high-disp \\
\hline 30406 & 08 Apr. 95 & $1.5 \mathrm{~min}$ & - & underexposed \\
\hline
\end{tabular}

1995. Since for the first IUE observations, few photometric and spectroscopic observations were available for this object, no precise estimation of the extinction and consequently of the exposure times could be made in advance. Optimum exposure times were obtained empirically after trial observations in both IUE cameras. All the data were obtained in the low dispersion mode $(\Delta \lambda \sim 6 \AA)$ in the long-wavelength (LWP) and short-wavelength (SWP) cameras through the large aperture $\left(10^{\prime \prime} \times 20^{\prime \prime}\right)$. We note that due to the size of the IUE entrance aperture, for all observations regardless of the aperture orientation, both stars (A and B) are expected to fall within this aperture, but due to brightness differences of the binary pair and the somewhat short exposures taken, HD 144432 A is possibly the largest contributor to the observed fluxes, although we cannot completely exclude the potential contribution of HD 144432 B, especially shortward of $1700 \AA$. Observations secured in 1995 , have also been retrieved from the archives. The LWP image 30393 is the only high-dispersion image taken on this object. A journal of the observations is provided in Table 1 .

\subsection{Near-Infrared spectroscopy}

Near-infrared (1.9-4.1 $\mu \mathrm{m})$ spectroscopy of the HD 144432 system was obtained on June 28, 2002, at Mauna Kea Observatory with the SpeX spectrograph (Rayner et al. 2003) attached to the IRTF. Since two sources were visible on the acquisition image, separate spectra were taken, of the southernmost, IR-brightest, component, HD $144432 \mathrm{~A}$, and the fainter, northernmost, of the two, HD 144432 B. During both observations, the slit, with a width of $0.8^{\prime \prime}$, was aligned East-West in order to minimize contamination of the individual spectra by the companion.

The near-infrared spectrum of HD $144432 \mathrm{~A}$ is dominated by strong emission lines of HI. Br $\alpha(4.05 \mu \mathrm{m})$, Pf $\gamma(3.74 \mu \mathrm{m})$, $\operatorname{Br} \gamma(2.17 \mu \mathrm{m})$, and $\operatorname{Br} \delta(1.94 \mu \mathrm{m})$ are clearly detected in Fig. 7. $\operatorname{Br} \beta(2.63 \mu \mathrm{m})$ is in the observed wavelength-range, but it is obscured by terrestrial atmospheric bands. The only clearly detected absorption lines in HD $144432 \mathrm{~A}$ are the $\Delta v=2$ bandheads of ${ }^{12} \mathrm{CO}$ at 2.32, 2.37 and $2.44 \mu \mathrm{m}$. The solid-state band at $3.3 \mu \mathrm{m}$ due to Polycyclic Aromatic Hydrocarbons (PAHS), often detected in HAeBe stars, is not present in the spectrum of
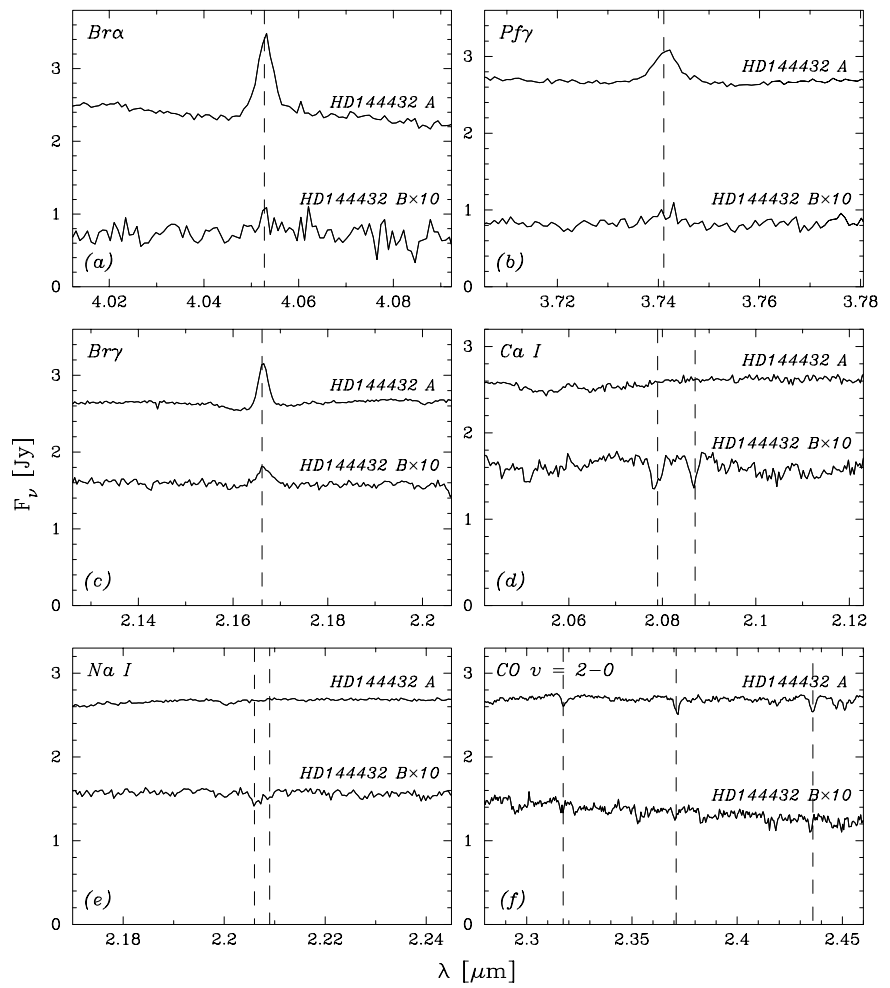

Fig. 7. Overview of near-infrared emission and absorption lines detected in HD $144432 \mathrm{~A}$ and B. a) The Brackett $\alpha$ line at $4.05 \mu \mathrm{m}$, b) Pfund $\gamma(3.74 \mu \mathrm{m}), \mathbf{c})$ Brackett $\gamma(2.17 \mu \mathrm{m})$, d) the Ca I lines at 2.08 and $2.09 \mu \mathrm{m}$, e) the Na I doublet at $2.21 \mu \mathrm{m}, \mathbf{f})$ the ${ }^{12} \mathrm{CO}$ complex at $2.29-2.46 \mu \mathrm{m}$. The top curve in each panel shows the HD $144432 \mathrm{~A}$ spectrum, whereas the bottom curve depicts the HD 144432 B spectrum, with the flux multiplied by 10 for visualisation. Dashed lines in each panel show the rest wavelength(s) of the depicted line(s). Note that for the $\mathrm{CO}$ lines, these dashed lines indicate the wavelength of the band-head; absorption in the ${ }^{12} \mathrm{CO}$ R-branch shortward of the bandhead wavelengths is present in HD $144432 \mathrm{~B}$.

HD 144432 A. We derive an upper limit of $4.0 \times 10^{-15} \mathrm{~W} \mathrm{~m}^{-2}$ for the flux of this feature in HD $144432 \mathrm{~A}$. We conclude that the near-infrared spectrum of HD 144432 A is clearly non-stellar in origin. We are probably detecting here the onset of the strong infrared excess due to circumstellar dust, prominently visible in the spectral energy distribution (SED) displayed in Fig. 8, with some emission and weaker absorption features superimposed due to a gaseous inner accretion disk.

In contrast to HD $144432 \mathrm{~A}$, the near-infrared spectrum of HD $144432 \mathrm{~B}$ is dominated by absorption lines that are probably photospheric in origin (Fig. 7). The Na I doublet at $2.21 \mu \mathrm{m}$, as well as the $\mathrm{Ca}$ I absorption lines at 1.978 and $1.987 \mu \mathrm{m}$, are clearly detected. The ${ }^{12} \mathrm{CO}$ R-branch transition of CO is weakly present in absorption. The only detected emission line is $\mathrm{Br} \gamma$ at $2.17 \mu \mathrm{m}$. The seeing at the time of observation, as well as the employed background-subtraction scheme is such that we can exclude that this is due to contamination of the point-source HD 144432 A seen in the $K$-band image. 


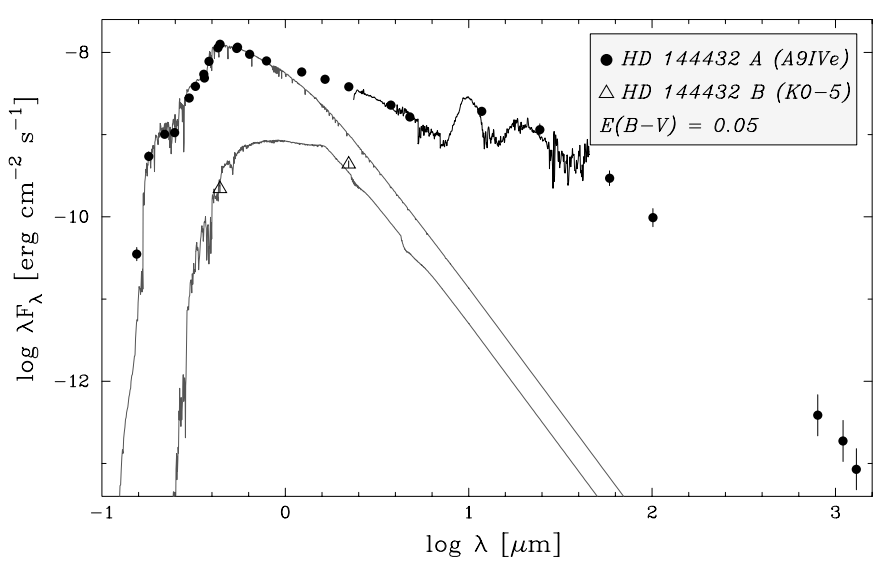

Fig. 8. Spectral energy distributions (SEDs) of HD 144432 A (filled dots) and HD 144432 B (open triangles). We also plot the ISO-SWS spectrum of HD 144432 described by Meeus et al. (2001). For both stars we also show a reddened Kurucz (1991) model stellar photosphere fitted to the SED. Note the strong infrared-mm wave excess above the photospheric fit for HD 144432 A. Also note the strong emission band around $10 \mu \mathrm{m}$ proving the presence of silicate dust.

\section{Data analysis}

\subsection{Variability}

The comparison of the SWP images 38425 and 39496 taken 5 months apart revealed virtually no variability in the continuum fluxes beyond the repeatability errors, therefore, for the rest of the analysis these images were coadded. The same is true for the LWP images 17585 and 17586, which were coadded and weighted according to their exposures times; the overexposed part of LWP 17585 was properly flagged and not considered in the final spectra. We warn that if the star is variable, the fact that no variability is detected in these images could be only a fortunate coincidence.

\subsection{FES magnitudes}

By using the IUE Fine Error Sensor (FES) counts recorded on the IUE observing scripts and the calibration by Pérez \& Loomis (1991), we were able to estimate $V$ magnitudes for the times at which the 1990 IUE exposures were taken at the NASA-GSFC Observatory. Since the 1995 exposures taken at the ESA-VILSPA Observatory, FES counts were affected by the abnormal increase in scattered light seen by the telescope optics (known as the FES anomaly detected on January 22, 1991) consequently no $V$ magnitudes were derived for these exposures. The $V(\mathrm{FES})$ magnitudes are listed in Table 1 . The differences in $V(\mathrm{FES})$ values are within the errors of this calibration $( \pm 0.03 \mathrm{mag})$ and no significance is attributed to the small discrepancies in the $V$ magnitudes. We note that the nonvariability of HD 144432 is in agreement with earlier photometric monitoring of HD 144432 by Meeus et al. (1998) and van den Ancker et al. (1998), but confirms this behaviour on a longer time-scale than reported in those papers.

\subsection{Spectral type}

When analyzing our visual spectrum of HD 144432 (see Fig. 2), it can be recognized as that of a star of late A or early F-type with emission lines. The spectrum is dominated by the Balmer lines. $\mathrm{H} \alpha$ shows strong emission, whereas all other Balmer lines, visible up to $\mathrm{H} 12$ are in absorption. The Ca II $\mathrm{K}$ line is moderately strong in absorption (the other line of this doublet, $\mathrm{Ca}$ II $\mathrm{H}$, is blended with $\mathrm{H} \varepsilon$ ), suggesting a spectral type of late A. However, the $G$ band at $4300 \AA$ is also definitely present, although not very strong, suggesting a slightly later spectral type of F0-2. The Na I doublet at $5885 \AA$ has strong emission components, suggesting that these lines may be similar in nature to those of HAeBe stars like UX Ori (e.g. Grinin et al. 1994; Grady et al. 1996). In addition to this, the area between 4150 and $6500 \AA$ shows many metallic absorption lines of Fe I, Fe II, Mg I and Mg II. O I may be present in absorption at 6161, 7777 and $8440 \AA$, but these could possibly also be attributed to other ions. At the red end of the spectrum the Paschen lines can be seen in absorption up to P20. Superimposed on P15 and P16 we can see two lines of the Ca triplet at 8500 and $8542 \AA$ in emission. The third line of this triplet, at $8665 \AA$, may be present in emission, but because it is blended with several lines of the Paschen series this is harder to distinguish.

The photometric catalog by Hauck \& Mermilliod (1990) gives a value of $\mathrm{H} \beta=2.730$ for $\mathrm{HD} 144432$, which corresponds to a star of spectral type F0-2 III-V according to the Strömgren calibration by Lester et al. (1986). However, by using the Kurucz (1991) models scaled at $V=8.30$, we show in Fig. 8 that the best fit for the unredenned optical data is for the model with $T_{\text {eff }}=7500 \mathrm{~K}, \log g=4.0$ and $[\mathrm{Fe} / \mathrm{H}]=0.0$ (thick line), by assuming a normal extinction law (i.e., $R_{V}=3.1$ ). According to the Schmidt-Kaler (1982) calibration of effective temperatures, this corresponds to a spectral type of A8 III-V. The main difference between the observed data and the Kurucz model is the absence of $\mathrm{Mg}$ II absorption in HD 144432, which is discussed later. The predicted continuum flux as derived from the Kurucz model shortward of $1700 \AA$, is virtually zero, whereas the stellar flux is significantly higher showing a moderate UV flux continuum and line excesses. In comparing with UV standard stars from Heck et al. (1984), the spectral types A8-9 are conspicuously absent, however, compared with HD 87696 (sp type: A7V), HD 144432 is probably a half or a full spectral type later.

A respectable previous estimate of the spectral type as A9/F0V is presented by Houk (1982) in the Michigan catalog of Two Dimensional Spectral Types for the HD Stars, Vol. 3. We note that no emissions or peculiarities are seen in the wavelength window of $3920-4900 \AA$ used for this classification. More recent spectral classifications of HD 144432 as A9/F0Ve (Dunkin et al. 1997), and A9IVe (Merín \& Montesinos 2000) confirm this spectral classification suggesting that the spectral type of HD 144432 appears constant with time. 


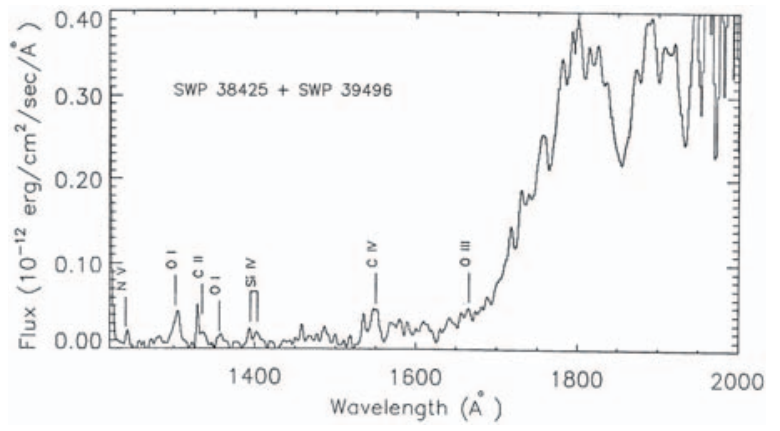

Fig. 9. Short wavelength (SWP) camera spectrum showing a large number of emissions not seen in main sequence stars; $\mathrm{NV}$, O I, O III, Si IV, C II, C IV, suggesting a superionization region of at least $8 \times 10^{5} \mathrm{~K}$.

\subsection{The emission spectra}

A detailed analysis of the line spectra of the coadded data for the SWP and LWP revealed the presence of interesting emissions.

\subsubsection{Short wavelengths $[1200-2000 \AA]$}

In Fig. 9 we identified the emission lines present such

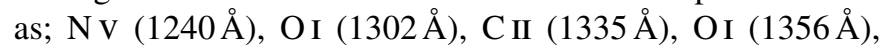
Si IV (1394, $1403 \AA$ ), C IV (1550 ̊), O III (1666 $)$. Certainly emissions at these wavelengths are not seen in main sequence stars, however, they are detectable in stars with active chromospheres and/or pre-main sequence objects. The star under consideration could have an incipient chromosphere, however, this kind of activity tends to peak at later spectral types. On the other hand, pre-main sequence stars such as HR 5999 (spectral type A5-7 III-IV), presents the same emission lines suggesting the presence of super-ionization regions capable of reaching temperatures of $\sim 8 \times 10^{5} \mathrm{~K}$. The origins of these emissions in the past have been interpreted in terms of chromospheric activity, even for the earlier types (early A- and B-type), however, recent evidences are accumulating which favor clumpy accretion, boundary layers and bipolar flows as the main mechanisms responsible for most of the excesses and emission lines in the optical and UV (Graham 1992; Welty et al. 1992; Pérez et al. 1993; Grady et al. 1993).

\subsubsection{Long wavelengths [2000-3200 Å]}

In this wavelength range we can distinguish several Fe II UV multiplets (mostly in absorption), which are typical in young and pre-main sequence objects. The circumstellar emission surrounding the central source is normally optically thick at UV wavelengths presenting itself as continuum flux heavily absorbed by strong Fe opacities. In HD 144432, the Fe II UV multiplets 1, 62, 63 and 64 appear in absorption, however, in a few HAeBes some Fe II lines also appear in emission. In addition, we can distinguish the emission at Mg II (UV1) and the absorption at $\mathrm{Mg}$ I (UV1). In a study of the behavior of the Mg II lines in HAeBes, Pérez et al. (1992) determined that the youngest and most extreme objects in the sample always
HD 144432 (LWP 30392, 63 min)

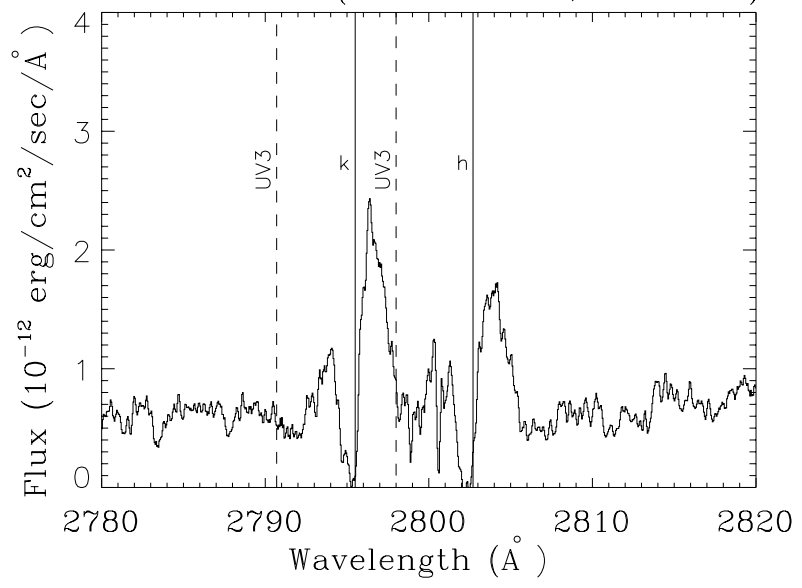

Fig. 10. The P Cygni profiles of the $\mathrm{Mg}$ II $\mathrm{h}$ and $\mathrm{k}$ lines. The Fe II lines (UV3) also are indicated.

presented Mg II lines ( $\mathrm{h}$ and $\mathrm{k}$ ) in emission, usually displaying direct P Cygni profiles, which often can only be resolved in high-dispersion $\left(R \sim 10^{4}\right)$ images. The excess flux detection in HD 144432 of the Mg II (2798 $\AA$ ) line from LWP images has also been reported by Valenti et al. (2003).

We also note that the resonance $\mathrm{Mg}$ II lines in emission are not unique to HAeBes and that a few stars such as B[e] stars, binaries, symbiotic stars and chromospherically active late-type stars also present this emission. The weak emission at Mg II as detected in low-dispersion, is a further corroboration that this object is likely to be young (note that classical Be and main sequence B stars generally present only $\mathrm{Mg}$ II - $\mathrm{h}$ and $\mathrm{k}$ - in absorption). Finally, the LWP high dispersion image, seen in Fig. 10, allows us to verify that the $\mathrm{Mg}$ II lines are indeed in emission with a well-defined P Cygni profile.

\subsection{Distance}

The distance towards HD 144432 is somewhat problematic. The distances quoted in the literature range from $108 \mathrm{pc}$ to $2.4 \mathrm{kpc}$. The near distance determination is based on a so-called "spectroscopic parallax", i.e. simply by assuming that the star is on the main-sequence, which is probably incorrect. From similar arguments, but assuming that HD 144432 is a postAGB star, Pottasch \& Parthasarathy (1988) conclude that the distance towards HD 144432 is $2.4 \mathrm{kpc}$.

HD 144432 is located in the region of the sky occupied by Sco OB2-2 $(d=145 \mathrm{pc})$, which would strongly indicate that it belongs to this group, suggesting a distance of about $145 \mathrm{pc}$. Two B2V stars, 13 Sco and $\rho$ Sco are a few degrees away from HD 144432 and both are members of the Sco OB2-2 association. The proper motions of all three stars agree suggesting that they all belong to Sco OB2-2.

HD 144432 was also measured by the Hipparcos satellite, but the parallax measurement only resulted in a $2.5 \sigma$ detection. However, its distance was derived to be $250 \mathrm{pc}$ (with a large error bar); inconsistent with the determination by Pottasch \& Parthasarathy (1988). Additional evidence for a near rather than a far distance are the weak interstellar Na I D-line (5890 ̊) 
shown by Meeus et al. (1998) and also observed by one of us (BWB). We conclude that the observed Na I D-line strength constrains the distance towards HD 144432 to be between 100 and $500 \mathrm{pc}$, ruling out the $2.4 \mathrm{kpc}$ distance derived by Pottasch \& Parthasarathy (1988).

Perhaps the best estimate for the true distance may come from an astrophysical argument. From the spectral energy distribution, one can compute a luminosity density, $L_{\star} / d^{2}$, of $4.9 \times 10^{-4} L_{\odot} \mathrm{pc}^{-2}$ for HD 144432. The luminosity for a A9 V star is $7.6 L_{\odot}$, which would translate to a distance of 124 pc. If we assume HD 144432 to be an A9 III giant, its luminosity should be around $23 L_{\odot}$, which would place it around $216 \mathrm{pc}$. So, if we assume that the spectroscopic classification of HD 144432 as A9 IV is correct, this would lead us to a distance smaller than $200 \mathrm{pc}$, which now contradicts the Hipparcos data. We note that the renewed realization that HD 144432 is a binary system also casts some doubt on the Hipparcos result, since an unrecognized binary motion in the system could affect the outcome of the solution for the parallax by the automated Hipparcos algorithm.

We conclude that HD 144432 is most likely a member of Sco OB2-2, with a distance of 145 pc. However, we warn about the discrepancy with Hipparcos parallax measurements, which for many other young objects have proven to be a powerful tool to ascertain their distances (van den Ancker et al. 1997, 1998).

\subsection{The companion}

A faint companion to HD 144432 was detected in the $K$-band $(2.2 \mu \mathrm{m})$ image of HD 144432, obtained at the IRTF (Fig. 1). As was already noted in Sect. 2.4, the infrared spectrum of the companion, HD $144432 \mathrm{~B}$, is dominated by absorption lines that are probably photospheric in origin. The ratio of the strength of the $2.21 \mu \mathrm{m} \mathrm{Na}$ I doublet to that of the $2.30 \mu \mathrm{m}$ $\mathrm{CO}$ feature has been shown to be a good indicator of spectral type in pre-main sequence stars (e.g. Doppmann \& Jaffe 2003). The fact that they appear to have nearly equal strength in HD 144432 B, suggests a spectral type of early-mid K for this star.

Our IRTF image shows that in June 2002, HD 144432 B was located $1.4^{\prime \prime}$ from the primary at a position angle of $-7^{\circ}$. A search of the SIMBAD database revealed that HD 144432 had been classified before as a close visual binary, Rossiter 1877 (Rossiter 1955). The 1934 measurement of this gave a separation of $1.2^{\prime \prime}$ with position angle of 354 degrees, and photographic magnitudes of 8.4 and 12.9 for the two components. This is essentially identical to the position of the companion seen at the IRTF. With the early-K spectral type derived from the IRTF spectrum, the photographic magnitude of 12.9 is also compatible with the brightness of HD $144432 \mathrm{~B}$ in the IRTF $K$-band image. Therefore, we conclude we are seeing the same companion, at nearly the same relative position, as was seen in 1934 in the optical.

We note that the proper motion of HD 144432 as measured by Hipparcos (30 milliarcseconds per year), means that in the course of the 68 years between the two measurements, the separation between the two should have changed

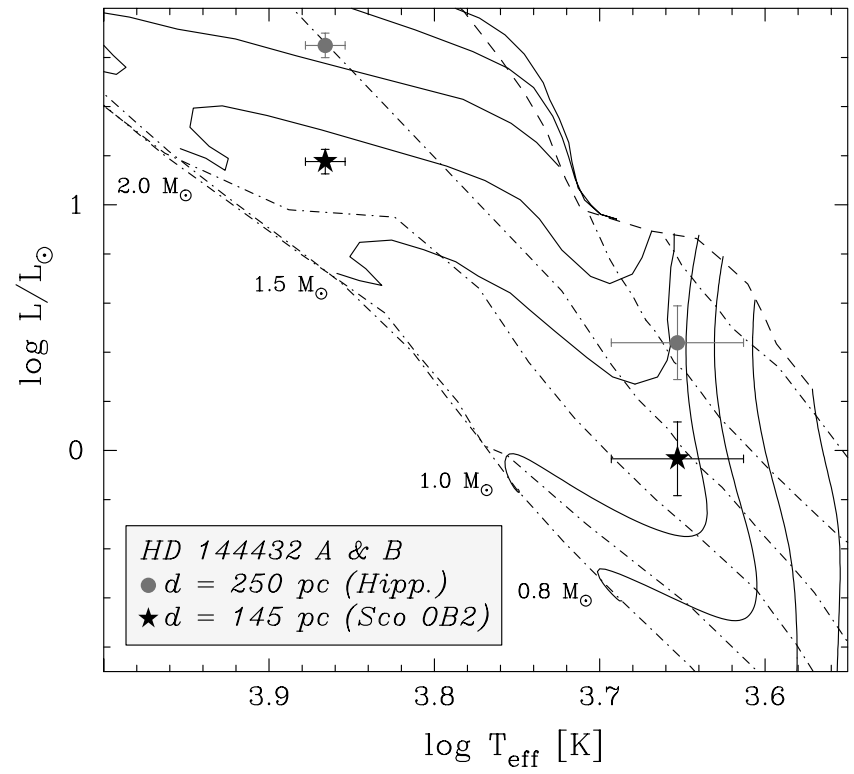

Fig. 11. Hertzsprung-Russell diagram for HD 144432 A and B for a common distance of $145 \mathrm{pc}$ (dots) and $250 \mathrm{pc}$ (stars). Also shown are the pre-main sequence evolutionary tracks (solid lines) and isochrones (dash-dotted) by Palla \& Stahler (1993).

by about $2.0^{\prime \prime}$ if the two components had different proper motions. This has apparently not happened. Consequently, we conclude that HD $144432 \mathrm{~B}$ shares a common proper motion with HD 144432 A, strongly suggesting a common distance and origin.

From the detailed SED of HD 144432 A (Fig. 8), we derive $L_{\star} / d^{2}$ of $7.1 \times 10^{-4} L_{\odot} \mathrm{pc}^{-2}$ for HD $144432 \mathrm{~A}$. The IRTF $K$ band magnitude of 8.3 for HD 144432 B translates to $L_{\star} / d^{2}=4.4 \times 10^{-5} L_{\odot} \mathrm{pc}^{-2}$ if we adopt a K2 spectral type and assume the (small) colour excess $E(B-V)$ for HD $144432 \mathrm{~A}$ and $\mathrm{B}$ to be the same. If we plot these values in a HR diagram for the two possible distances of 145 and 250 pc (Fig. 11), we note that for both distances, HD 144432 A and B would be located above the main sequence, in an area typically occupied by pre-main sequence stars. Although we cannot exclude the $250 \mathrm{pc}$ distance completely, we note that for the $145 \mathrm{pc}$ distance both HD $144432 \mathrm{~A}$ and B are located between the isochrones for 1 and 3 million years, whereas for the $250 \mathrm{pc}$, a small discrepancy in age between the two systems would exist.

\section{Conclusions}

Our observations have demonstrated that HD 144432 is indeed a complex and intriguing object. We confirm its pre-main sequence nature, and have verified the existence of a faint latetype companion to HD 144432. The common proper motion and age of HD 144432 A and B strongly suggest that they have formed together. Further research is needed to investigate whether this companion, as HD 144432 A itself, is still surrounded by significant amounts of circumstellar material, or whether it has already shed most of its natal cloud.

The detection of Li I (6708 $⿱$ A) absorption in the integrated spectrum of HD 144432 is intriguing. The simple-minded explanation that this could form in the atmosphere of the late-type 
star HD 144432 B is problematic, since the Li I line presents the same rotational broadening (see Fig. 4) as the metal lines. Unless the low-mass binary companion has a similar rotational broadening as the primary, which we consider unlikely, this strongly suggests that Li I is produced in the same stellar photosphere, i.e., intrinsic to HD 144432 A. Early F-type stars can present the Li I absorption, but at this photospheric temperature $\mathrm{Li}$ is mostly in the form of Li II, so our measured $E W(\mathrm{Li}$ I) of 93 mA would imply an usual high Li abundance in HD 144432.

The UV observations of HD 144432 also confirmed the youth of this star, as a probable pre-main sequence object. The UV spectral type of this star appears to be earlier than the optical type. The trend of earlier spectral types with decreasing wavelength is a clear indication of the inconsistency of the single-temperature stellar model, which has been found to be the hallmark of young objects such as FU Orionis (Hartmann \& Kenyon 1985) and other HAeBe stars (Pérez et al. 1993, and references therein). This effect has been interpreted as evidence for the presence of emission from an optically thick accretion disk and boundary layer, rather than from a stellar photosphere. The presence of several emission lines shortward of $1700 \AA$, species commonly associated with chromospheres in late-type stars, suggests that these emissions probably have a more energetic origin such as the one predicted by the accretion disk + boundary layer model for HAeBe stars (Hillenbrand et al. 1992; Blondel et al. 1993). Corroborating evidence for the youth of this object are its physical location in the upper Scorpius region and the large infrared excess indicating the existence of large amount of circumstellar material capable of thermally re-emitting at longer wavelengths.

Acknowledgements. This research made use of the SIMBAD database, operated at CDS, Strasbourg, France. We are grateful to Dr. Fancis Fekel for obtaining spectra at $6430 \AA$ (Fig. 3) at our request. We are especially grateful to the referee of this manuscript, Dr. Claude Catala, for the several suggestions that resulted in many clarifications and improvements to this paper. We also acknowledge the financial assistance of the Los Alamos Laboratory-Directed Research and Development (LDRD) used to complete work included in this paper (LA-UR-03-6569).

\section{References}

Blondel, P. F. C., Talavera, A., Tjin, A., \& Djie, H. R. E. 1993, A\&A, 268,624

Carballo, R., Wesselius, P. R., \& Whittet, D. C. B. 1992, A\&A, 262, 106

Catala, C., Czarny, J., Felenbok, P., \& Praderie, F. 1986, A\&A, 154, 103

Corporon, P., \& Lagrange, A. M. 1999, A\&AS, 136, 429

Dunkin, S. K., Barlow, M. J., \& Ryan, S. G. 1997, MNRAS, 286, 604

Doppmann, G. W., \& Jaffe, D. T. 2003, AJ, 126, 3030

Grady, C. A., Pérez, M. R., \& Thé, P. S. 1993, A\&A, 274, 847
Grady, C. A., Pérez, M. R., Talavera, A., et al. 1996, A\&AS, 120, 157

Graham, J. A. 1992, PASP, 104, 479

Grinin, V.P ., Thé, P. S., de Winter, D., et al. 1994, A\&A, 292, 165

Hamann, F., \& Persson, S. E. 1992, ApJS, 82, 285

Hauck, B., \& Mermilliod, J. C. 1990, A\&AS, 86, 107

Hartmann, L., \& Kenyon, S. J. 1985, ApJ, 299, 462

Houk, N. 1982, Catalogue of two-dimensional spectral types for the HD stars, 3, Michigan Spectral Survey, Ann Arbor, Dept. of Astronomy, Univ. of Michigan

Heck, A., Egret, D., Jaschek, M., \& Jaschek, C. 1984, IUE LowDispersion Spectra Reference Atlas- Part 1. Normal Stars. ESA SP-1052

Herbig, G. H. 1960, ApJS, 4, 337

Hillenbrand, L. A., Strom, S. E., Vrba, F. J., \& Keene, J. 1992, ApJ, 397,613

Jaschek, M., Jaschek, C., \& Andrillat, Y. 1988, A\&AS, 72, 505

Jaschek, M., Jaschek, C., \& Andrillat, Y. 1991, A\&A, 250, 127

Kurucz, R. L. 1991, in Precision Photometry: Astrophysics of the Galaxy, ed. A. G. Davis Philip, A. R. Upgren, \& K. A. Janes (Schenectady, N.Y., L. Davis Press)

Lester, J. B., Gray, R. O., \& Kurucz R. L. 1986, ApJS, 61, 509

Maheswar, G., Manoj, P., \& Bhatt, H. C. 2002, A\&A, 387, 1003

Meeus, G., Waelkens, C., \& Malfait, K. 1998, A\&A, 329, 131

Meeus, G., Waters, L. B. F. M., Bouwman, J., et al. 2001, A\&A, 365, 476

Merín, B., \& Montesinos, B. 2000, ASP Conf. Ser., 219, 399

Oudmaijer, R. D., van der Veen, W. E. C. J., Waters, L. B. F. M., et al. 1992, A\&AS, 96, 625

Palla, F., \& Stahler, S. W. 1993, ApJ, 418, 414

Pérez, M. R., \& Loomis, C. 1991, Record of the IUE Three Agency Coordination Meeting (NASA/ESA/SERC), Nov 19-21, 1991, GSFC, F-13

Pérez, M. R., Imhoff, C. L., \& Thé, P. S. 1992, BAAS, 23, 1374

Pérez, M. R., Grady, C. A., \& Thé, P. S. 1993, A\&A, 274, 381

Pottasch, S. R., Parthasarathy, M. 1988, A\&A, 192, 182

Rayner, J. T., Toomey, D. W., Onaka, P. M., et al. 2003, PASP, 155, 362

Rossiter, R. A. 1955, Publ. Michigan Obs., 11, 1

Schmidt-Kaler, Th. 1982, in Landolt-Börnstein Tables, New Series (Berlin and New York: Springer-Verlag), Group VI, 2

Soderblom, D. R., Jones, B. F., Balachandran, S., et al. 1993, ApJ, 106, 1059

Sylvester, R. J., Barlow, M. J., \& Skinner, C. J. 1995, ApSS, 224, 405

Suchkov, A. A., Schultz, A. B., \& Lisse, C. M. 2002, ApJ, 570, L29

Thé, P. S, de Winter, D., \& Pérez, M. R. 1994, A\&AS 104, 315

van Boekel, R., Waters, L. B. F. M., Dominik, C., et al. 2003, A\&A, 400, L21

van den Ancker, M. E., Thé, P. S., Tjin A Djie, H. R. E., et al. 1997, A\&A, 324, L33

van den Ancker, M. E., de Winter, D., \& Tjin A Djie, H. R. E. 1998, A\&A, 330, 145

Valenti, J. A., Fallong, A. A., \& Johns-Krull, C. M. 2003, ApJS, 147, 305

Wackerling, L. R. 1970, Mem. RAS, 73, 153

Walker, H. J., \& Butner, H. M. 1995, ApSS, 224, 389

Walker, H. E., \& Wolstencroft, R. D. 1988, PASP, 100, 1509

Welty, A. S., Strom, S. E., Edwards, S., Kenyon, S. J., \& Hartmann, L. W. 1992, ApJ, 397, 260

Zuckerman, B., Forveille, T., \& Kastner, J. H. 1995, Nature, 373, 494 